\title{
El informe de auditoría en los países del MILA
}

The auditor's report in the MILA countries

\author{
Julián Zamarra-Londoño \\ Universidad de Antioquia, Medellín, Colombia. \\ Daniela Pérez-Norela iD \\ Universidad de Antioquia, Medellín, Colombia. \\ Aura Pareja-Taborda \\ Universidad de Antioquia, Medellín, Colombia.
}

\begin{abstract}
Resumen
Objetivo: Realizar una revisión de los informes de auditoría de las Compañías de los países pertenecientes al Mercado Integrado Latinoamericano-MILA (Colombia, Perú, Chile y México), respecto al cumplimiento de los aspectos que se deben considerar según las Normas Internacionales de Aseguramiento de la Información para el periodo 2010 a 2018. Método: investigación de carácter cualitativa, mediante análisis documental realizado a los párrafos de énfasis, de otros asuntos y de cuestiones clave. Resultados: se encontró que el cumplimiento de la norma ha sido satisfactorio, en especial en lo referente a cuestiones adicionales de información, se observa que se ha aplicado las normas, incluso antes de la obligatoriedad que se tiene para los países estudiados. Discusiones: las llamadas BIG4 son las firmas que mayor número de párrafos de énfasis, otros asuntos y cuestiones claves emiten, haciendo referencia principalmente a la adopción de normas, políticas contables y bases para la opinión. Conclusiones: las cuestiones adicionales que se revelan en el informe de auditoría podrían tener un efecto en la toma de decisiones de los usuarios ya que revela información adicional para los usuarios de la información.
\end{abstract}

Palabras clave: Dictamen de auditoria, información financiera, norma internacional de auditoría, normatividad contable.

Clasificación JEL: M42

Autor de Correspondencia

julian.zamarra@udea.edu.co

Recibido: $15-12-2019$

Aceptado: 12-03-2020

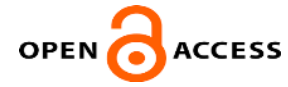

(cc) BY

Copyright (c) 2020

Desarrollo Gerencial

\section{Abstract}

Purpose: To review the auditor's reports issued by the companies based in the countries that comprise the Latin American Integrated Market (MILA, in the Spanish acronym) [Colombia, Peru, Chile, and Mexico] regarding compliance with the International Auditing and Assurance Standards for 20102018. Method: Qualitative research by carrying out the documentary analysis of emphasis of matter paragraphs, other matter paragraphs, and key audit matters. Results: Standard compliance was satisfactory. Standards regarding additional information were applied, even before such application was mandatory in the countries researched. Discussions: BIG 4 firms issue the highest number of emphasis of matter paragraphs, other matter paragraphs, and key audit matters, referring mainly to the adoption of standards, accounting policies, and basis for opinion. Conclusions: The other elements disclosed in the auditor's report may affect users' decision-making processes since they provide information users with additional information.

Keywords: auditor's opinion, financial information, international auditing standards, accounting requirements.

JEL Classification: M42

Como citar este artículo (APA):

Zamarra-Londoño, J., Pérez-Norela, D., \& Pareja-Taborda, A. (2020). El informe de auditoría en los países del MILA. Desarrollo

Gerencial, 12(1), 1-20. https://doi.org/10.17081/dege.12.1.3783 


\section{Introducción}

El dictamen de auditoría es el resultado del trabajo que evidencia la responsabilidad que tiene el auditor de planear y realizar la auditoría para obtener una seguridad razonable y objetiva acerca de si los estados financieros están libres de errores materiales. El informe de auditoría es entonces un documento que emite un auditor externo en el que expresa su opinión acerca de la razonabilidad de dichos estados financieros que, de acuerdo con un marco normativo contable tiene la pretensión de aumentar el grado de confianza para los diferentes usuarios de la información.

Para llevar a cabo el proceso de la auditoría de estados financieros el auditor verifica el cumplimiento de la preparación de dichos estados financieros bajo los requisitos establecidos respecto de todo lo material expresados en las Normas de Auditoria Internacionales (International Auditing and Assurance Standards Board IAASB, 2013). Sin embargo, el dictamen no solo comprende la emisión de una opinión, sino que también debe reflejar otros elementos dentro del informe como son: otras explicaciones que brindan información adicional a los usuarios de la información para tomar decisiones respecto a posibles inversiones en las compañías, dicha información adicional se podría ver reflejada en las cuestiones adicionales que revela el auditor bajo la International Standard on Auditing 701 (2013a) e International Standard on Auditing 706 (2013b) respecto a párrafos de énfasis, de otros asuntos y de cuestiones claves, que tienen por objetivo revelar mayor información para los usuario.

En este sentido, estas revelaciones deberían constituirse como una herramienta de gran valor en la toma de decisiones, entendiendo la importancia de la razonabilidad de la información financiera y considerando que no solo debe tenerse en cuenta la opinión del auditor, sino que adicionalmente puede contener otro tipo de información relevante y de utilidad en el proceso decisorio de los usuarios de la información. Este informe emitido por el auditor debería cobrar mayor relevancia en el contexto del mercado de valores, en tanto que allí el contacto entre los inversionistas y las empresas se ve materializado en muchas ocasiones por medio de comunicación del informe de auditoría, dichos mercados se vuelven relevantes en el contexto de la auditoria, en la medida que estos se estructuran bajo dinámicas que parten de la búsqueda de la eficiencia y la maximización de beneficios para las partes involucradas en las diversas transacciones (Al-Thuneibat, Khamees \& Al-Fayoumi, 2008).

De esta manera, toma relevancia hacer una revisión de los dictámenes de las empresas pertenecientes al Mercado Integrado Latino Americano (MILA), el cual es una unión de mercados bursátiles en el que se transan títulos valores de cuatro países latinoamericanos, teniendo en cuenta que este es una integración de mercado compuesta por varios países en los que la normatividad contable puede tener variaciones entre sí, pero con un común denominador en cuanto a la adopción (aún en diferentes momentos) de las 
Julián Zamarra Londoño, Daniela Pérez Norela, Aura Pareja Taborda

Normas Internacionales de Auditoría (NIA) y que, por ende, en la actualidad incorporan el mismo referente normativo para realizar y establecer el párrafo de énfasis, párrafo de otros asuntos y/o cuestiones claves en el dictamen, basados en la Norma Internacional de Auditoría-NIA 706 (2013b) aplicable en todos los países que hacen parte de este estudio (Colombia, Perú, Chile y México),

Si bien es cierto que el dictamen tiene unos elementos generales y unos requisitos mínimos señalados por las NIA para los países mencionado, el mismo puede expresar situaciones relevantes para que el usuario identifique cuáles son los hechos que llamaron su atención e incluso pudieron generar incertidumbres sobre la información proporcionada y por ello que cobra importancia identificar este tipo de revelaciones en los dictámenes y poder identificar si se encuentran elementos comunes o que puedan ser tendencia dentro de los países estudiados.

Considerando entonces que las revelaciones adicionales mencionadas anteriormente, influyen e intervienen en la toma de decisiones de los usuarios de la información frente a las compañías, resulta relevante estudiar el nivel de cumplimiento de tales párrafos con la norma reglamentada en la NIA 706 y NIA 701, con el objetivo de evaluar el contenido de los párrafos de énfasis, otros asuntos y párrafos de cuestiones claves de las empresas auditadas en el MILA entre 2010 y 2018, además de evaluar cuales empresas cumplen con los aspectos requeridos en normatividad, cuáles son los temas revelados en estos y los factores existentes más relevantes y cuáles de ellos tienen en común las empresas analizadas.

Dado lo anterior, en la siguiente sección, se realiza una descripción del contenido del informe de auditoría y la influencia de particularidades de cada país en el mismo, debido a -entre otras cosas-, la transición normativa en materia del aseguramiento de la información que derivó en la sustitución de las normas locales por los estándares internacionales. Luego, se presentan los procedimientos metodológicos adoptados en la investigación y la descripción del análisis de datos. Al final del trabajo, se hacen algunas consideraciones sobre la evidencia encontrada en este estudio.

\section{Fundamentación teórica}

\section{El dictamen de auditoría}

La auditoría es un proceso llevado a cabo dentro de las organizaciones como herramienta de control y supervisión, que permite detectar fallas o errores dentro de las estructuras de la organización (Villardefrancos y Riviera, 2006); este proceso es realizado por una persona independiente y competente, que esté en la capacidad de evaluar la información de la compañía, además de que funge como el responsable de certificar la información presentada por la compañía auditada (Arens \& Loebbecke, 1980). Lo anterior, denota el papel de control que ejerce la auditoría en las organizaciones, encaminado al correcto que hacer de las operaciones y finanzas de las mismas, dando muestra de su importancia en 


\section{Julián Zamarra Londoño, Daniela Pérez Norela, Aura Pareja Taborda}

tanto que permite "verificar que la información financiera, administrativa y operacional que se genera, es confiable, veraz y oportuna" (Morales, 2012, p. 12), convirtiéndose en garantía general para la comprobación de que los hechos económicos se realizan de forma adecuada y que las políticas y controles se estén realizando acorde con lo estipulado en la normatividad vigente de cada país, permitiendo analizar la forma en la que se está administrando los recursos de una compañía.

Como se mencionó anteriormente, la auditoría es ejecutada por lo general por una persona independiente, en empresas de mediano y gran tamaño, son firmas de auditoría las que se encargan de estos procesos (Estefanell \& Fusté, 1980). Así, dichas empresas tienen como respectivo soporte del trabajo de auditoria el informe que se emite al final del trabajo y que constituye la opinión sobre la razonabilidad de la información presentada en los estados financieros, de manera tal que en dicho informe el auditor da cumplimiento a sus responsabilidades, fundamentando su opinión en las conclusiones concebidas a través del trabajo de auditoría efectuado y compilado en la evidencia obtenida de la empresa (IAASB, 2013).

Ahora bien, desde las disposiciones de los estándares internacionales de aseguramiento de la información, el propósito del informe sirve como medio de comunicación entre el auditor y los diferentes usuarios y este debe incluir: el título, el destinatario, un apartado introductorio, la responsabilidad de la dirección con relación a los estados financieros, la responsabilidad del auditor, la opinión del auditor sobre los estados financieros, la firma y dirección del auditor, y la fecha de emisión del informe (IAASB, 2013; Salehi \& Abedini, 2008). Además, en caso de considerarlo necesario, el auditor incluirá dentro de su informe otra información que sea relevante para llamar la atención de los usuarios sobre un asunto determinado que le permita a estos últimos obtener un mayor grado de comprensión de los estados financieros o de la auditoría; tal revelación la hará por medio de los párrafos de énfasis, de otros asuntos y de cuestiones claves.

Por consiguiente, dado el rol que toma la auditoría entre las organizaciones y los usuarios, diversas investigaciones han pretendido establecer el impacto que tiene el reporte del auditor en los usuarios de la información, dado que la opinión allí contenida les permite adquirir un grado de confianza razonable frente a la información suministrada por las entidades (Abdullah, 2017). Adicionalmente, el que este informe esté preparado bajo estándares comunes a nivel global, le otorga credibilidad a la información financiera frente a inversionistas extranjeros con desconocimiento de la normativa local de los países donde operan las entidades permitiéndoles mayor entendimiento del negocio (Haliti, Ismajli \& Mustafa, 2017).

Por otro lado, el informe del auditor sirve como medio de revelación de la capacidad de las entidades para continuar como negocio en marcha, facultando a los usuarios para actuar de acuerdo a la percepción que estos tengan sobre la probabilidad de si sus intereses se vean afectados por las distintas situaciones 
Julián Zamarra Londoño, Daniela Pérez Norela, Aura Pareja Taborda

que puedan presentarse al interior de las organizaciones (Cha, Hwang \& Yeo, 2016), lo cual hace que el impacto de este informe se extienda más allá de las organizaciones, pues deriva de las acciones emprendidas por los usuarios, variables como el precio de la acción se ven modificadas (Hoti, Ismajli, Ahmeti \& Dërmaku, 2012) (Robu \& Robu, 2015).

\section{El párrafo de énfasis, párrafo de otros asuntos y párrafos de cuestiones claves en el dictamen de auditoría}

Algunos autores como Coram, Mock, Turner \& Gray (2011) han señalado que la percepción de los usuarios frente al informe de auditoría está orientada a que el mismo no reviste mayor importancia más allá de contener la opinión relativa a la razonabilidad de los estados financieros con relación a un marco de información financiera, ocasionando que el mismo no posea valor para fundamentar un proceso de análisis; sin embargo, el informe de auditoría, además de contener la opinión del auditor que de por sí es fundamental para el proceso de la toma de decisiones sobre información que ha sido asegurada y verificada, regularmente incluye información adicional como los párrafos de énfasis, otros asuntos y/o cuestiones claves, por lo que recientemente han sido diversos los autores que se han interesado por evaluar tal contenido incluido en los informes de auditoría y determinar cuál es el impacto que el mismo genera en los diferentes usuarios a los que esté dirigido.

Ahora bien, si se consideran dichos párrafos como los componentes del dictamen de auditoría donde se presenta asuntos que a consideración del auditor son relevantes tanto para efectos de los estados financieros de la compañía como para la toma de decisiones de los usuarios, estos deben ser reglamentados, puesto que tienen un alto efecto sobre las decisiones. Sin embargo, la existencia de los párrafos en un dictamen de auditoría no siempre significa la presencia de alguna salvedad a consideración del auditor, ya que en muchas ocasiones estos párrafos se centran específicamente en un tema de interés puntual, casi siempre relacionado con las notas a los estados financieros y que a su juicio es importante resaltarlos, pero sin afectar su opinión.

Por lo anterior, en los países latinoamericanos se adoptan las Normas Internacionales de Auditoría emitidas por el Consejo de Normas Internacionales de Auditoría y Aseguramiento (IAASB por su siglas en inglés, International Auditing and Assurance Standards Board), en tales normas se regulan las circunstancias en que el auditor puede incluir un párrafo de énfasis, de otros asuntos o de cuestiones claves, cuyos aspectos deben quedar expuestos en una nota a los estados financieros y además brindar una clara comunicación adicional en el informe de auditoría (Fonseca, 2009).

Por su parte, Spathis (2003), menciona que los párrafos incluidos en el dictamen deben informar no solo situaciones relacionadas con las notas a los estados financieros, sino asuntos críticos de auditoría donde se informe a inversionistas y los diferentes usuarios en general, de alguna posible discontinuidad 


\section{Julián Zamarra Londoño, Daniela Pérez Norela, Aura Pareja Taborda}

en las operaciones, dejando claro a los interesados de la información de una compañía, la incertidumbre sobre sus inversiones y el posible riesgo en la asignación de los recursos financieros de los inversores, dado las afectaciones que puede tener dichos párrafos, en consecuencia tiene un impacto grande en el mercado, dado que la información allí presentada a juicio del auditor afecta la toma de decisiones de inversores y demás usuarios externos de la información financiera de la compañía.

Asimismo, Dutran, Alberton \& Van (2007), menciona que los párrafos adicionales en los dictámenes de auditoría constituyen elementos relevantes para un análisis financiero integral de los mismos y contribuyen al análisis de escenarios de incertidumbre organizacional. Lo anterior da pie a que sea de gran importancia determinar entonces si dichos párrafos se realizan de acuerdo con la normatividad correspondiente, puesto que, si de alguna forma se incluye información que no se establece, se pierde las características de énfasis.

\section{Mercado Integrado latinoamericano (MILA) y adopción de Normas Internacionales de Auditoría}

El dictamen de auditoría y las cuestiones adicionales que se refieren anteriormente tienen gran relevancia en los países y además en integraciones de mercado en la medida que brindan información adicional a los inversionistas para tomar decisiones. Por esto mismo, es referente para el estudio la integración de los mercados bursátiles MILA, como asociación de cuatro países que realizaron un acuerdo en la reducción y eliminación de barreras comerciales en los respectivos mercados, es decir, que tienen privilegios y acceso fácil a los mercados del acuerdo. Lo anterior, busca eliminar barreras de comercio que, de acuerdo con Enderlein (2011) y Corbo (1997), aumenta la liberalización y comercio de los mercados financieros que han llevado de forma creciente a una expansión de los flujos financieros entre países de la región y afuera de esta.

En el caso de Latinoamérica, se presenta el Mercado Integrado Latinoamericano (MILA) que es en principio la alianza entre 3 bolsas de valores, la Bolsa de Comercio de Santiago, la Bolsa de Valores de Colombia y la Bolsa de Valores de Lima, así como de los depósitos DECEVAL, DCV y CAVALI, las cuales, desde 2009, iniciaron el proceso de creación de un mercado regional para la negociación de títulos de renta variable de los tres países, e iniciaron operaciones en Mayo de 2011 (MILA, 2018) y a finales del 2014 se unió la Bolsa de Valores de México (Lancheros, 2017) para ser hoy por hoy la integración de 4 países; cada bolsa de cada país sigue siendo independiente $y$, por ende, tiene características propias $y$, posiblemente, niveles de eficiencia y de estacionalidades diferentes (Worthington \& Higgs, 2006).

De esta integración se evidencian diferentes beneficios para los inversionistas tales como: el mejoramiento del balance riesgo-retorno y la disposición de más instrumentos financieros para diversas operaciones de compra y venta, a los emisores les permite tener un mayor acceso a nuevos mercados y 


\section{Julián Zamarra Londoño, Daniela Pérez Norela, Aura Pareja Taborda}

un aumento en la demanda de los activos emitidos. También es importante mencionar que, si bien se esperan beneficios de la integración de estos mercados, también existen dificultades o desafíos que podrían obstaculizar la consolidación del MILA, teniendo diferencias regulativas en los mercados de valores de los diferentes países (Vasgas y Bayardo, 2013).

Hablando específicamente del Mercado Integrado Latinoamericano (MILA) y los párrafos de énfasis, de otros asuntos y cuestiones claves en el dictamen de auditoría toma importancia debido al impacto que genera en la regulación de los países que lo integran. En cada uno de los países que conforman esta integración como lo son Chile, Colombia, México y Perú se cuenta con normatividad específica para regular dichos párrafos ya que es un aspecto clave del informe de auditoría, que puede generar impactos positivos y negativos en la toma de decisiones de los usuarios de la información financiera respecto a las empresas y como consecuencia puede derivar en una afectación a la economía de cada país que integran este mercado. Algunos de los impactos positivos que se pueden desprender de la presencia de los párrafos de énfasis de otros asuntos y cuestiones claves en el dictamen de auditoría es la valoración de activos y de la empresa como tal, debido a lo manifestado por parte del auditor independiente, pero por otra parte, el párrafo de énfasis puede significar que los inversores se desincentiven de realizar inversiones o tomar decisiones acerca de las inversiones ya existentes en las empresa, al igual que los demás usuarios de la información financiera pueden verse afectados a la hora de la toma de decisiones como consecuencia no de la opinión del auditor ya que la misma no puede verse afectada por lo manifestado en dichos párrafos.

En la Tabla 1 se evidencian algunas características de los países pertenecientes al MILA, las cuales son de gran importancia, ellas son las denominaciones de los aseguradores de la información financiera y la clasificación de las normas para cada país.

Tabla 1

Referente normativo Normas Internacionales de Auditoría en el Mercado Integrado Latinoamericano
Características
Colombia
Perú
Chile
México

Adopción de NIA

A partir del año

2016

Diciembre de 2006

Diciembre de 2012

1 de enero de

2012

Denominaciones

Aseguradores de la

Información financiera

Revisor Fiscal

Consejo de vigilancia

Auditor Externo-

Inspector de Cuentas

Comisario

Ley 43 de 1990 y las Normas de Auditoría

Antes de la adopción de NIA, ley que aplicaba.
Generalmente

Aceptadas en

Colombia - NAGA
Ley 28.708 es la ley general del Sistema de Contabilidad Nacional
Elaborado con base en las normas emitidas por el AICPA (AICPA, 2017).
Normas de

Auditoría Generalmente Aceptadas (NAGA) 
Julián Zamarra Londoño, Daniela Pérez Norela, Aura Pareja Taborda

\begin{tabular}{|c|c|c|c|c|}
\hline Clasificación NAGAS & $\begin{array}{l}\text { Normas generales o } \\
\text { personales, Normas } \\
\text { de ejecución del } \\
\text { trabajo, Normas y } \\
\text { preparación del } \\
\text { informe }\end{array}$ & $\begin{array}{l}\text { Normas generales o } \\
\text { personales, Normas de } \\
\text { ejecución del trabajo, Normas } \\
\text { y preparación del informe }\end{array}$ & $\begin{array}{l}\text { Se agrupa en } \\
\text { sección AU }\end{array}$ & $\begin{array}{l}\text { Se agrupa en } \\
\text { boletines }\end{array}$ \\
\hline $\begin{array}{l}\text { Supervisión y Miembro } \\
\text { de la IFAC y aprobación }\end{array}$ & $\begin{array}{l}\text { PCAOB (The Public } \\
\text { Company } \\
\text { Accounting } \\
\text { Oversight } \\
\text { Board) Empresas } \\
\text { que cotizan en bolsa }\end{array}$ & $\begin{array}{l}\text { (JDCCPP / Junta de decanos } \\
\text { de los Colegios de Contadores } \\
\text { Públicos de Perú) }\end{array}$ & $\begin{array}{l}\text { Colegio de } \\
\text { Contadores de Chile } \\
\text { (CCCH) }\end{array}$ & $\begin{array}{l}\text { Comité Ejecutivo } \\
\text { Nacional del } \\
\text { Instituto Mexicano } \\
\text { de Contadores } \\
\text { Público }\end{array}$ \\
\hline
\end{tabular}

Fuente: elaboración propia (2019).

\section{Antecedentes en cuanto a revelaciones adicionales del Informe de Auditoría}

En cuanto a lo que otros autores han estudiado respecto a la problemática en cuestión mencionada en los apartados anteriores, se destacan algunas estudios como por ejemplo el realizado por Cevallos (2016) donde hace un análisis y clasificación de los párrafos de énfasis del dictamen de auditoría emitido por el auditor, donde se consideran diferentes factores que influyen a la hora de la presentación de los párrafos de énfasis, algunas de estas clasificaciones son la forma jurídica o tamaño de la empresa, variando así la presentación de los párrafos de énfasis de acuerdo a la empresa, sector o características especiales consideradas en la planeación del trabajo de auditoría. Adicionalmente, como mencionan Damascena y Paulo (2013), los párrafos de énfasis emitidos por los auditores se ven influenciados por factores externos como lo son los indicadores financieros o el valor de mercado de la compañía, donde las variables financieras pueden causar un impacto sobre la opinión que emite el auditor y a su vez la magnitud de la influencia de esta opinión sobre los usuarios y/o inversores de la compañía, sin embargo no existe una clara relación de dichos párrafos de énfasis u opinión de auditoría respecto al cumplimiento legal de los mismos ya sea tratándose de su contenido o estructura.

Por ende, de acuerdo con los referentes anteriores se puede identificar que los estudios de los párrafos de énfasis se desarrollan en torno a su estructura y tipos de opiniones que pueden contener de acuerdo con la empresa, sector o demás factores que sean relevantes según el juicio del auditor. Sin embargo, autores como Da Silva, Benevides y Nobrega (2014), se centran en la normativa vigente y en su cumplimiento, analizando la normativa y regulación relacionada con las opiniones emitidas en los dictámenes, sus exigencias, y su cumplimiento e incumplimiento por parte de algunas compañías brasileras. Por otro lado, en la Tabla 2 se muestran otros referentes acerca de investigaciones relacionadas. 


\section{Tabla 2}

\section{Referentes teóricos sobre las comunicaciones adicionales del dictamen de auditoría}

Autor

Descripción

(Dutran, Alberton

\& Van (2007)

(Damascena,

Firmino \& Paulo, 2011)

(Damascena \&

Paulo, 2013)

(Rumitti y Gómez, 2017)

(Sirois, Bédard, \& Bera, 2018)

(Seaton \& Montague, 2018)
Los autores analizan la adherencia normativa de la emisión de los dictámenes de los auditores independientes en relación con la normativa vigente brasileña NBC T11, específicamente en los párrafos de énfasis contenidos en los mismos. La investigación fue de carácter exploratorio, teóricoempírica mediante encuestas. Se tiene que se ciñen de manera satisfactoria la muestra de 136 dictámenes analizados en los periodos de 1999 a 2006, sin embargo aunque el nivel de cumplimiento es alto, se identifican cuestiones ambiguas en relación a la presentación del párrafo de énfasis ya que hay existencia de hechos que generan incertidumbre a la situación de la empresa en particular, la presentación de este párrafo sin dicha incertidumbre significara entonces que pierde sus características y se limite a ser la mera presentación de información adicional relevante.

Mediante una investigación descriptiva y bibliográfica, los autores analizaron los asuntos más comunes que dan origen a la presentación de salvedades y/o de un párrafo de énfasis en el informe de auditoría, tomando para esto una muestra de 1.466 informes de los cuales el $44 \%$ contaba con párrafos de énfasis o alguna salvedad. Se identificó que en relación a la existencia de párrafos de énfasis en el informe de auditoría se tienen 10 situaciones comunes que dan origen a párrafos de énfasis como lo son: perdidas recurrentes, patrimonio líquido negativo, deficiencia de capital, discusiones judiciales, créditos tributarios, cuentas de difícil cobro, activos y pasivos sujetos a modificación judicial, reposicionamiento tarifario con posibles efectos en el patrimonio, falta de examen de auditoría en el ejercicio actual o anterior.

Analizan como las variables contables e indicadores financieros de las empresas que cotizan en la bolsa de valores de Sao Paulo generan una influencia significativa en la opinión del auditor y en que exista o no párrafo de énfasis en el dictamen de auditoría, desarrollado esto a través de análisis estadístico LOGIT para una muestra de 1466 dictámenes. Obteniendo como resultado que hay mayor probabilidad a contar con párrafo de énfasis en sus dictámenes.

Las modificaciones introducidas en las Normas Internacionales de Auditoría (NIA) requieren cambios sustanciales en la estructura del Informe. Incorporan nuevas secciones: "Asuntos clave de Auditoría" y "Otra información"; modificaciones en su distribución y variaciones sobre la empresa en marcha. A pesar de los objetivos pretendidos, quedan dudas sobre la posibilidad de que todos los usuarios de los estados contables puedan comprender el informe. Parece dificultosa su aplicación en entes y estudios de auditoría de pequeñas estructuras, significando una carga adicional de dudosas ventajas.

En este estudio, se buscó identificar el impacto de la existencia de párrafos de énfasis y Key audit matters (KAM) en la atención de los usuarios en el informe de auditoría y en la información financiera de las empresas, mediante una metodología experimental aplicando la tecnología de seguimiento ocular se analizó la atención o el detalle con que abordan los usuarios los informes de auditoría. El resultado de la investigación arrojó que cuando se presenta un párrafo de énfasis en el dictamen de auditoría la atención del usuario se centra allí y entre más asuntos existan en el dictamen se va perdiendo atención a los demás asuntos de la información financiera, lo cual muestra el impacto que tienen los párrafos de énfasis o asuntos críticos de auditoría en el dictamen ya que se convierten en el centro de atención con el fin de tomar decisiones acertadas.

Se analiza la influencia de los párrafos de énfasis en la toma de decisiones los inversionistas debido a la importancia de la manifestación de incertidumbre en los informes de auditoría, mediante una metodología experimental donde se evidencia que la existencia de párrafos de énfasis puede afectar la probabilidad de inversión, mientras que si no existen dichos párrafos de énfasis la propensión a invertir es mayor, igualmente si los párrafos de énfasis se encuentran de manera amplia en el informe de auditoría se determina un efecto negativo en relación a la toma de decisiones de los inversionistas. 


\section{Método}

\section{Diseño}

Este estudio parte de una investigación de enfoque cualitativo, con alcance descriptivo y en el cual se utilizó técnicas como el análisis documental, con el fin de realizar una revisión de los informes de auditoría de las Compañías de los países pertenecientes al Mercado Integrado Latinoamericano-MILA (Colombia, Perú, Chile y México), respecto al cumplimiento de los aspectos que se deben considerar según las Normas Internacionales de Aseguramiento de la Información (IAASB) en el contenido de los párrafos de énfasis, otros asuntos y cuestiones claves de auditoría para el periodo 2010 a 2018.

\section{Participantes}

La unidad de análisis la conforman las empresas que pertenecen al Mercado Integrado Latinoamericano (MILA), con las cuales se realizó un análisis de la revelación en párrafos de énfasis de otros asuntos y/o cuestiones claves en los países pertenecientes a este mercado, partiendo de una población de 452 empresas del MILA equivalentes a 4770 observaciones, las cuales se filtraron de acuerdo con el estado de cada una de ellas, así se tuvieron en cuenta las empresas que estaban activas y listadas en la bolsa de valores de cada país objeto de estudio, además de acuerdo con la disponibilidad de información del dictamen de auditoria, obteniéndose de esta forma un resultado de 20 empresas de Colombia, 57 de Perú, 178 de Chile y 35 empresas mexicanas, para un periodo de análisis de 2010 a 2018 y una muestra total de 290 empresas por año de estudio.

\section{Instrumentos}

Se utilizó una matriz de análisis de contenido enfocado en las características de cuestiones adicionales reveladas en el informe de auditoría como: tipo de dictamen, firma de auditoría, párrafo de énfasis, tema del énfasis, objetivo del párrafo, párrafo de otros asuntos, número de otros asuntos, tema de este, cuestiones claves (KAM), número de KAM, temas de chequeo de cumplimiento de la normatividad. Los datos para la construcción de la matriz se extrajeron de la lectura de los informes de auditoría de las empresas seleccionadas para el periodo 2010-2018, los cuales se diligenciaron de forma manual en la matriz. La fuente de los informes fueron las páginas web de las empresas de la muestra y páginas de las bolsas de valores que contenían información adicional.

\section{Resultados}

Los resultados, que pueden verse en la Tabla 3, muestran que del total de empresas analizadas (según la información obtenida de los informes de auditoría) para Colombia el número de informes que contienen 


\section{Julián Zamarra Londoño, Daniela Pérez Norela, Aura Pareja Taborda}

párrafo de énfasis son 49 y párrafo de otros asuntos es de 153 desde el año de aplicación de la norma comprendidos entre el año 2016 y 2018; para Perú el número de informes que contienen párrafo de énfasis después del año de aplicación de la norma comprendidos entre el año 2016 y 2018 son 157 y el número de informes que contiene párrafo de otros asuntos es de 95, para el tema de cuestiones claves solo México cuenta con información, mientras que Colombia, Chile Perú aún no aplican dicha normativa.

\section{Tabla 3}

Número de informes que contienen párrafo énfasis de otros asuntos y/o cuestiones calves

$\begin{array}{cccc}\text { PAÍS } & \begin{array}{c}\text { Número de informes que } \\ \text { contienen párrafo de } \\ \text { énfasis }\end{array} & \begin{array}{c}\text { Número de informes } \\ \text { que contienen párrafo } \\ \text { de otros asuntos }\end{array} & \begin{array}{c}\text { Número de informes que } \\ \text { contienen párrafo de } \\ \text { cuestiones claves }\end{array} \\ \text { COLOMBIA } & 49 & 153 & 0 \\ \text { PERÚ } & 157 & 95 & 0 \\ \text { MÉXICO } & 25 & 130 & 310 \\ \text { CHILE } & 435 & 315 & 0\end{array}$

Fuente: elaboración propia (2019).

Ahora bien, si se enfoca en el análisis del el caso particular de México a partir de su periodo de aplicación (año 2014), de los informes publicados correspondientes a 35 empresas de 2014 a 2018, como se evidencia en la Tabla 3, 25 informes contienen párrafos de énfasis, 130 incluyen párrafos de otros asuntos y 310 contienen cuestiones claves de auditoría, del total de los reportes en solo 40, el auditor no aclara la posición del auditor frente a la situación que se resalta. Para el caso de Chile, la norma aplica a partir de diciembre de 2014, por lo cual se realizó el análisis de los resultados desde dicho año, según la información obtenida de los informes publicados correspondientes a 178 empresas durante 2014 a 2018, 435 informes contienen párrafos de énfasis y 315 incluyen párrafos de otros asuntos, de los informes que contienen tanto párrafos de énfasis como de otros asuntos solo 392 aclaran la posición del auditor frente a la situación que se resalta.

Por otro lado, desde el año de aplicación de las NIA para los países pertenecientes al MILA, se evidencia que en cada país hay una utilización diferente de los párrafos, por ejemplo, en el caso de Colombia las empresas hacen mayor uso del párrafo de otros asuntos; sin embargo, no hay distinción de la norma en estos párrafos ya que en esta se especifica que debe contener cada párrafo y en las empresas de este país se homogeniza la información revelada. En Perú, se identifica que la información adicional que se revela no tiene una distinción entre aquella revelada entre el párrafo de énfasis y el párrafo de otros asuntos. En México se revela información adicional en el párrafo de cuestiones claves en los que se explican cada cuestión y se abarca también el trabajo realizado por el auditor en cada una estas, en Chile desde el año de aplicación, las empresas revelan la información adicional por medio del párrafo de énfasis, se observa que las empresas en sus informes los auditores hicieron un correcto uso del párrafo ya que en su mayoría la información está de acuerdo al marco normativo. 
En cuanto, a la información que revelan en los informes de auditoría y las firmas de auditorías, predominan emisión de cuestiones adicionales las denominadas Big 4, (Big Four, término en inglés) como se observa en la Tabla 4 en la que se muestran las firmas de auditorías para cada uno de los países en estudio. La mayoría de las firmas revisoras son: Deloitte, PriceWaterhouseCooper, KPMG y Ernst and Young. Para el caso de Colombia se nota que tienen total participación para emisión de párrafos de énfasis y otros asuntos y si bien para emisión de dictamen también se encuentra un porcentaje de no Big, estas no realizan revelación de cuestiones adicionales o no aplican la norma en cuestión; Para Perú estas grandes firmas tienen participación en las empresas por medio de firmas de auditoría locales como lo son Gris y Asociados, Gaveglio Aparicio y Asociados, Caipo y Asociados, Paredes Zoldiver burga y asociados, entre otras. Por otra parte, las empresas que no son consideradas Big 4 tienen baja participación en países como Perú, estas empresas son locales y trabajan bajo su propio nombre; para el caso de México y Chile prepondera la participación las grandes firmas. Sin embargo, las pequeñas empresas de auditoras también están haciendo presencia en el mercado.

\section{Tabla 4}

\section{Emisión de párrafos de énfasis por tipo de firma}

$\begin{array}{ccc}\text { PAIS } & \text { BIG } 4 & \text { NO BIG } 4 \\ \text { COLOMBIA } & 77 & 0 \\ \text { PERÚ } & 391 & 52 \\ \text { MÉXICO } & 426 & 71 \\ \text { CHILE } & 653 & 136\end{array}$

Fuente: elaboración propia (2019).

Además, en el caso particular de la información revelada por las empresas chilenas con relación a las firmas de auditoría mediante las cuales se realiza la emisión de los informes, el $83 \%$ es respaldado por las Big 4, y el $17 \%$ restante se encuentra centralizados entre RSM Chile, Nattero y CIA Ltda, Suriatina Auditores Ltda y SMS Latinoamérica. En México más del $86 \%$ de los informes emitidos se concentra en las $B I G 4$, y en el nicho restante se encuentran firmas independientes como Russell Bedford, Organización Descom S.C., Meza M. Figueroa y Asociados S.C., JHI, DFK International, Grant Thornton, Moore Stephens, PKF, Crowe Horwath International, BDO, Vacco y Guzmán Tello de Meneses, S.C., de las cuales Grant Thornton tiene mayor participación seguida de Moore Stephens.

Como se puede evidenciar en la Tabla 4 y en lo descrito anteriormente, las firmas Big 4 tiene mayor participación en la información que se revela en relación con los párrafo de énfasis, adicional, al evaluar la información que presentan se identifica que dicha información presenta una tendencia homogénea, es decir, que esta información se repite según la firma que realice el proceso de auditoría en las empresas, dentro de los temas de los que más se revela información de las empresas en los párrafo se tiene notas 
Julián Zamarra Londoño, Daniela Pérez Norela, Aura Pareja Taborda

anexas para el análisis de los EEFF, método en que revelan el valor de sus inversiones, adopción de normas contables, modificación de estatutos, reexpresión y ajuste e Estados financieros, hechos económicos, fenómenos societarios y cambios en políticas contables, adicional, en lo referente a las cuestiones claves solo México ha iniciado la implementación de este asunto en sus informes de auditoría, relacionándolo principalmente con cuestiones claves de hechos económicos de contingencias y provisiones (Tabla 5).

Tabla 5

Asuntos predominantes en los informes de auditoría

\begin{tabular}{|c|c|c|c|c|}
\hline PAIS & $\begin{array}{c}\text { Tema predominante } \\
\text { énfasis }\end{array}$ & Subtema & $\begin{array}{l}\text { Tema predominante } \\
\text { otros asuntos }\end{array}$ & Subtema \\
\hline MÉXICO & $\begin{array}{l}\text { Normas y políticas } \\
\text { contables }\end{array}$ & $\begin{array}{l}\text { Adopción de la } \\
\text { norma }\end{array}$ & Bases para la opinión & $\begin{array}{c}\text { Información no } \\
\text { auditada/Otra } \\
\text { información }\end{array}$ \\
\hline CHILE & Hechos económicos & $\begin{array}{l}\text { Registros de } \\
\text { operación }\end{array}$ & Antecedentes Histórica & $\begin{array}{c}\text { Información de } \\
\text { auditores predecesores }\end{array}$ \\
\hline COLOMBIA & Hechos económicos & Información de EF & $\begin{array}{l}\text { Normas y políticas } \\
\text { contables }\end{array}$ & Información de EF \\
\hline PERÚ & Hechos económicos & $\begin{array}{l}\text { Operaciones y } \\
\text { ajustes a los EF }\end{array}$ & $\begin{array}{l}\text { Normas y políticas } \\
\text { contables }\end{array}$ & Adopción de normas \\
\hline
\end{tabular}

Adicional a lo anterior, se realizó un análisis del promedio de asuntos revelados en el informe de auditoría de los países pertenecientes al MILA, con el fin de identificar la tendencia promedio del mismo (Tabla 6).

\section{Tabla 6}

Promedio de asuntos mencionados por párrafo.

\begin{tabular}{|c|c|c|c|}
\hline PAIS & $\begin{array}{l}\text { Párrafo de otros } \\
\text { asuntos }\end{array}$ & Párrafo de énfasis & $\begin{array}{c}\text { Párrafo de cuestiones claves de } \\
\text { auditoría }\end{array}$ \\
\hline COLOMBIA & 2 & 3 & 0 \\
\hline PERÚ & 2 & 3 & 0 \\
\hline MÉXICO & 1 & 1 & 1 \\
\hline CHILE & 1 & 1 & 0 \\
\hline
\end{tabular}

El número de asuntos mencionados en los párrafos, partiendo de un promedio de asuntos revelados en los dictámenes por año, como se evidencia en la Tabla 6 para Colombia y Perú es de 2 asuntos en el párrafo de otras cuestiones $u$ otros asuntos y 3 asuntos mencionados en el párrafo de énfasis; los temas antes mencionados en la información que se revela en algunas empresas son los mismos para todos los años que se analizan. Para el caso de Chile y México se tiene que el número promedio de asuntos mencionados tanto en párrafos de énfasis como en otros asuntos es de 1 . Sin embargo, cabe aclarar que 
existen empresas que no presentan párrafos de énfasis, otros asuntos o cuestiones claves, y otras que presentan hasta 5 y 6 asuntos.

Ahora bien, con el fin de evaluar el cumplimiento de la norma para la presentación de los párrafos en el dictamen de auditoría según la NIA 706, se analizaron los requerimientos para cuando el auditor incluya un párrafo de énfasis en el informe de auditoría. Dicho párrafo se inserta inmediatamente después del párrafo de opinión y el párrafo de otros asuntos o de otras cuestiones se sitúa después del párrafo de énfasis o en caso de no revelar información en el párrafo de énfasis éste se sitúa después del párrafo de opinión. Teniendo en cuenta esto se hizo un análisis de los informes de auditoría (Tabla 7) donde de acuerdo a la información analizada en los informes se cumple en su gran mayoría con la norma: en el caso de Colombia, del total de empresas que en sus informes de auditoría incluyen párrafo de otros asuntos solo el $1,03 \%$ no presentan dicho párrafo en el orden en que lo especifica la norma y para Perú las empresas cumplen con la norma en su totalidad; el cumplimiento de la presentación del párrafo de énfasis en Colombia se cumple para todos los dictámenes y en Perú solo el 0,29\% no cumple con la norma, también se tiene que la aplicación de la norma antes de la obligatoriedad, dado que las empresas emitían información por medio de los párrafos de énfasis y otros asuntos pero el porcentaje de incumplimiento era mayor. Para Perú del total de empresas que en sus dictámenes de auditoría incluyen párrafo de énfasis en los periodos revelados el 0,59\% no incluyen notas anexas para el análisis de los estados financieros como lo especifica la norma.

Tabla 7

\section{Presentación de los párrafos}

\begin{tabular}{|c|c|c|c|c|c|c|c|c|}
\hline \multirow[t]{2}{*}{ PAIS } & \multicolumn{2}{|c|}{$\begin{array}{c}\text { ¿Está presentado el párrafo } \\
\text { de otros asuntos después } \\
\text { del párrafo de opinión y del } \\
\text { párrafo de énfasis? }\end{array}$} & \multicolumn{2}{|c|}{$\begin{array}{c}\text { ¿Está presentado el párrafo } \\
\text { de énfasis después del } \\
\text { párrafo de opinión? }\end{array}$} & \multicolumn{2}{|c|}{$\begin{array}{l}\text { ¿Se incluyen notas anexas } \\
\text { para el análisis de las } \\
\text { EEFF? }\end{array}$} & \multicolumn{2}{|c|}{$\begin{array}{c}\text { ¿Se incluyen en el párrafo } \\
\text { de énfasis notas } \\
\text { aclaratorias de los EEFF? }\end{array}$} \\
\hline & $\begin{array}{c}\% \\
\text { cumplimiento }\end{array}$ & $\begin{array}{c}\% \text { No } \\
\text { cumplimiento }\end{array}$ & $\begin{array}{c}\% \\
\text { cumplimiento }\end{array}$ & $\begin{array}{c}\% \text { No } \\
\text { cumplimiento }\end{array}$ & $\begin{array}{c}\% \\
\text { cumplimiento }\end{array}$ & $\begin{array}{c}\text { \%No } \\
\text { cumplimiento }\end{array}$ & $\begin{array}{c}\% \\
\text { cumplimiento }\end{array}$ & $\begin{array}{c}\text { \%No } \\
\text { cumplimiento }\end{array}$ \\
\hline COLOMBIA & $98,97 \%$ & $1,03 \%$ & $100,00 \%$ & $0 \%$ & $100,00 \%$ & $0 \%$ & $100,00 \%$ & $0 \%$ \\
\hline PERÚ & $100,00 \%$ & $0,00 \%$ & $99,71 \%$ & $0,29 \%$ & $99,41 \%$ & $0,59 \%$ & $99,71 \%$ & $0,29 \%$ \\
\hline MÉXICO & $97,69 \%$ & $2,31 \%$ & $99,92 \%$ & $0,08 \%$ & $99,88 \%$ & $0,12 \%$ & $100,00 \%$ & $0 \%$ \\
\hline CHILE & $99,05 \%$ & $0,95 \%$ & $98,62 \%$ & $1,38 \%$ & $96,09 \%$ & $3,91 \%$ & $100,00 \%$ & $0 \%$ \\
\hline
\end{tabular}

Por otra parte, haciendo referencia a los resultados obtenidos, para Chile en su mayoría todas las empresas que incluyen ya sea párrafo de énfasis o párrafo de otros asuntos cumplen con la norma, ya que el porcentaje de empresas donde el resultado en relación con la norma fue desfavorable oscila entre el $0.9 \%$ y el $3.9 \%$, que en promedio indica que tan solo el $2.07 \%$ de los informes en cuestión no siguen los lineamientos de la norma, la información revelada en el dictamen de auditoría por las empresas analizadas cambia a partir del año de aplicación obligatoria que es 2016 ya que en los años anteriores se incluía información relevante en el dictamen sin diferenciarse con el título, a partir del 2016 las empresas 


\section{Julián Zamarra Londoño, Daniela Pérez Norela, Aura Pareja Taborda}

tuvieron en cuenta los requisitos de la norma para la revelación de información importante. Para el caso de Chile y México sigue las mismas tendencias anteriores, dado el cumplimiento de la norma a partir la aplicación es alto y el porcentaje de incumplimiento oscila entre $1 \%$ y $4 \%$ en cada uno de los ítems analizados.

\section{Discusiones}

En torno a las cuestiones adicionales reveladas en el informe de auditoría Coram et. al (2011) resalta la importancia de que a través de éstas, los usuarios de la información adquieren una percepción de los resultados del proceso de auditoría través del informe; sin embargo, no se revela en concreto información más allá de la opinión relativa a la razonabilidad de los estados financieros, así mismo, los párrafos de énfasis, otros asuntos y cuestiones claves de auditoria manifestados en el dictamen no solo deben informar sobre elementos de las notas a los estados financieros, sino asuntos críticos de auditoría donde se informe a inversionistas y los diferentes usuarios en general, de alguna posible discontinuidad en las operaciones (Spathis, 2003). Lo anterior posee una relación con los resultados obtenidos en el presente estudio, en la medida que si bien se revela información adicional, gran parte de ella hace referencia solo a situaciones relacionadas con los estados financieros y no se revelan asuntos de riesgos o de la responsabilidad que tiene el auditor en el proceso de auditoría, limitando entonces el informe de auditoría solo a la opinión y no revelando información adicional necesaria para quien tiene por ejemplo la intención de invertir en la entidad.

La emisión de cuestiones adicionales que se revelan en el informe de auditoría, por ser regulados en la gran mayoría de los casos genera una adherencia satisfactoria, Dutra, Alberton \& Van (2007) expresan en su escrito que las empresas estudiadas se ciñen de manera satisfactoria a los párrafos de énfasis y el cumplimiento de los estándares es alto, situación similar presentan las empresas que pertenecen al MILA de acuerdo con los resultados obtenidos de la presente investigación donde el cumplimiento de la NIA 701 y 706 es significativo.

Según Damascena, Firmino \& Paulo (2011), las temáticas más abordadas con relación a las revelaciones de cuestiones adicionales tienen que ver con la existencia de pasivos vencidos y la deficiencia de capital, perdidas recurrentes, patrimonio líquido negativo, discusiones judiciales, créditos tributarios, cuentas de difícil cobro, activos y pasivos sujetos a modificación judicial, reposicionamiento tarifario con posibles efectos en el patrimonio, falta de examen de auditoría en el ejercicio actual o anterior. Para el caso del MILA se presentan algunos de los énfasis mencionados, se expresan significativamente temáticas relacionadas con elementos de normas y políticas contables en relación a adopción de normas y registros, ajustes de estados financieros, información no auditada e información de auditores predecesores. 
Los párrafos de énfasis, otros asuntos y cuestiones claves, como lo evaluó Damascena \& Paulo (2013), son mayormente emitidos por firmas de auditoría más pequeñas que emiten más informes con reservas y párrafos de énfasis, que las empresas de auditoría grandes llamadas BIG4, en el caso de la evaluación de presentación de revelaciones adicionales en MILA, se da el caso contrario, en la medida que las empresas que más revelan dicha información son las BIG4, esto se da en gran medida por la concentración del mercado de auditoria en estos países.

\section{Conclusiones}

El cumplimiento de la norma en las empresas analizadas ha sido asertivo, todas han adoptado las normas incluso antes de la adopción obligatoria del país correspondiente que, en todo caso, están alineadas con las NIA. Por su parte, se observa una participación mayoritaria de las firmas consideradas Big 4 en las empresas de los cuatro países pertenecientes al Mercado Integrado Latinoamericano -MILA en cuanto a la emisión de informes de auditoría al igual que del contenido de estos párrafos, y precisamente por ello mismo son estas firmas quienes mayor número de párrafos de énfasis, otros asuntos y cuestiones claves emiten. En cuanto a los párrafos de énfasis, de otros asuntos y de cuestiones claves, estos son utilizados por más del $50 \%$ de las empresas analizadas para revelar información adicional, considerada como la información que tiene efecto en la toma de decisiones de los usuarios y que a consideración de la auditoría y de su importancia relativa debe ser presentada en el informe.

El hecho de que más del $50 \%$ de las empresas analizadas utilicen los párrafos de énfasis, otros asuntos y cuestiones claves para enunciar los aspectos señalados en los resultados, es una clara evidencia que el informe del auditor por la estructura que inicialmente tiene antes de incorporarse los Key audit matters (KAM) tiene la necesidad de presentar más cantidad de información para que los usuarios del dictamen puedan entender y tomar decisiones con un mayor contenido de información. Lo que significa que con la incorporación en pleno de la NIA que introduce los KAM se podrá satisfacer en un mayor nivel las necesidades de los usuarios de información adicional.

Por otro lado, al estudiar la información contenida en los párrafos se concluye que los asuntos sobre los cuales se hace referencia en los dictámenes influyen en gran medida factores como la firma de auditoría que realice el proceso de auditoría ya que se evidencia que las firmas revelan información homogénea en las empresas en las que realizan los procesos de aseguramiento de la información. Se identificó la alta regulación que existe con la emisión de estos asuntos, ya que de las empresas estudiadas la mayoría cumple con los lineamientos de la norma al emitir dichos informes de auditoría que incluyen ya sea párrafo de énfasis, otros asuntos o cuestiones claves de auditoría. Finalmente se concluye que en países

como México en el caso particular de la aplicación de la NIA 701 "Cuestiones claves de auditoría", aunque la norma regía a partir del año 2017, muchas empresas mexicanas pertenecientes al MILA comenzaron a 
Julián Zamarra Londoño, Daniela Pérez Norela, Aura Pareja Taborda

revelar información de este tipo desde el año 2016. Lo anterior demuestra iniciativas de buen gobierno y estrategias de actualización continuada en los procesos de aseguramiento de las firmas auditoras para los países que hicieron parte de esta investigación.

\section{Referencias}

American Institute of Certified Public Accountants AICPA. (2017). Statements on Auditing Standards. https://www.aicpa.org/research/standards/auditattest/clarifiedsas.html

Abdullah, K. (2017). The Impact of Auditing on Stock Prices of Amman Stock Market's Listed Companies. International Journal of Academic Research in Business and Social Sciences, 76), 210-220. http://dx.doi.org/10.6007/IJARBSS/v7-i6/2958

Al-Thuneibat, A. A., Khamees, B. A., \& Al-Fayoumi, N. A. (2008). The effect of qualified auditors' opinions on share prices: evidence from Jordan. Managerial Auditing Journal, 23(1), 84-101. https://doi.org/10.1108/02686900810838182

Arens, A., \& Loebbecke, J. (1980). Auditing: An Integrated Approach (2a. ed.) New York: Prentice Hall.

Rumitti.C. y Gómez, M. (2017). Nuevas y revisadas Normas Internacionales de Auditoría. "Praestant magis quam certis captarem..." FACES, 23(49), 9-31. http://nulan.mdp.edu.ar/2761/

Cevallos, M. (2016). Un estudio descriptivo sobre la naturaleza de la opinión de auditoría en la región de Murcia [Tesis de Maestría, Universidad Politécnica de Cartagena] Repositorio Digital UPCT. http://hdl.handle.net/10317/5726

Cha, M., Hwang, K., \& Yeo, Y. (2016). Relationship between Audit Opinion and Credit Rating: Evidence from Korea. The Journal of Applied Business Research, 32(2), 621-634. https://doi.org/10.19030/jabr.v32i2.9599

Coram, P., Mock, T., Turner, J., \& Gray, G. (2011). The Communicative Value of the Auditor's Report. Australian Accounting Review, 21(3), 235-252. https://doi.org/10.1111/j.1835-2561.2011.00140.x

Corbo, V. (1997). Integración financiera en América latina [Documentos de trabajo de la oficina de economista jefe]. Banco Interamericano de Desarrollo. https://publications.iadb.org/es/publicacion/13353/integracion-financiera-en-america-latina 
Da Silva, E., Benevides, M., \& Nobrega, K. (2014). Um Enfoque sobre o Conteúdo Informacional dos Parágrafos de Ênfases e de outros Assuntos no Relatório dos Auditores Independentes de Companhias NA BM\&FBOVESPA. Revista de Administração, Contabilidade e Sustentabilidade, 4(3), 2041. https://doi.org/10.18696/reunir.v4i3.168

Damascena, L., Firmino, J., \& Paulo, E. (2011). Estudo sobre os pareceres de auditoria: análise dos parágrafos de ênfase e ressalvas constantes nas demonstrações contábeis das companhias listadas na Bovespa. 22(2), 125-154. https://revistas.face.ufmg.br/index.php/contabilidadevistaerevista/article/view/939

Damascena, L. \& Paulo, E. (2013). Pareceres de auditoria: um estudo das ressalvas e parágrafos. Universo Contábil, $\quad 9(3), \quad$ 104-127. https://gorila.furb.br/ojs/index.php/universocontabil/article/view/3321/2402

Dutran, A., Alberton, L., \& Van, B. (2007, 22-26 de septiembre). A Análise de Conteúdo aplicada aos Parágrafos de 'Ênfase' e de 'Informação Relevante' dos Pareceres da Auditoria Independente emitidos para as empresas do Setor Elétrico [conferencia]. Rio de Janeiro. http://www.anpad.org.br/diversos/down_zips/33/CON-A1172.pdf

Enderlein, H. (2011). The economic impact of the Deutsche Borse-Nyse Euronex Merger on the European financial markets. Berlin: Hertie School of Governance. http://www.hertieschool.org/enderlein/mergerstudy/

Estefanell, P., y Fusté, J. (1980). Objetivo y alcance de los trabajos realizados por firmas de auditores independientes. Revista española de financiación y contabilidad, IX(31), 49-64. https://www.aeca.es/old/pub/refc/n31.html

Fonseca, O. (2009). Dictámenes de Auditoría. Lima: IICO, Instituto de investigación en contabilidad y control.

Haliti, L., Ismajli, H., \& Mustafa, A. (2017). The importance of the audited financial statement in attracting foreign investors in private sector in Kosovo. Baltic Journal of Real Estate Economics and Construction Management, 5(1), 118-136. https://doi.org/10.1515/bjreecm-2017-0009 
Hoti, A., Ismajli, H., Ahmeti, S., \& Dërmaku, A. (2012). Effects of Audit Opinion on Stock Prices: The case of Croatia and Slovenia. EuroEconomica, 2(31), 75-87. http://journals.univdanubius.ro/index.php/euroeconomica/article/view/1276/1154

International Auditing and Assurance Standards Board IAASB. (2013). Manual de pronunciamientos internacionales de control de calidad, auditoría, revisión, otros encargos de aseguramiento y servicios relacionados partes I y II [Traducido al español de Federación Argentina de Consejos Profesionales de Ciencias Económicas, Instituto de Censores Jurados de Cuentas de España, Instituto Mexicano de Contadores Públicos, A.C.] Nueva York: Federación Nacional de Contadores (IFAC). https://www.iaasb.org/publications/manual-de-normas-internacionales-de-control-de-calidad-auditorrevisi-n-otros-encargos-de-8

International Auditing and Assurance Standards Board IAASB. (2013a). International Standard on Auditing 701: communicating key audit matters in the independent auditor's report. In IFAC (Ed.) Handbook of International Quality Control, Auditing Review, Other Assurance, and Related Services Pronouncements (pp. 1-15). Nueva York: The International Federation of Accountants (IFAC).https://www.iaasb.org/publications/reporting-audited-financial-statements-proposed-new-andrevised-international-standards-auditing

International Auditing and Assurance Standards Board IAASB. (2013b). International Standard on Auditing 706: emphasis of matter paragraphs and other matter paragraphs in the independent auditor's report. In IFAC (Ed.) Handbook of International Quality Control, Auditing Review, Other Assurance, and Related Services Pronouncements (pp. 713-723). Nueva York: The International Federation of Accountants (IFAC). https://www.iaasb.org/publications/international-standard-auditing-isa-706revised-emphasis-matter-paragraphs-and-other-matter-4

Lancheros, J. (2017). Análisis del proceso de integración del mercado latinoamericano (MILA) desde el 2011 al 2016 [Trabajo de pregrado, Corporación Universitaria iberoamericana] Repositorio Institucional-IBERO. https://repositorio.ibero.edu.co/handle/001/707/browse?locale=en

MILA (2018). Reseña histórica. https://mercadomila.com/quienes-somos/resena-historica/

Morales, H. (2012). Evolución histórica de la auditoría. En Morales (Ed.) Introducción a la auditoria (pp. 5136). México: Red Tercer Milenio S.C. 
Robu, M., \& Robu, I. (2015). The influence of the audit report on the relevance of accounting information reported by listed Romanian companies. Procedia Economics and Finance, (20), 562-570. https://doi.org/10.1016/S2212-5671(15)00109-4

Salehi, M., \& Abedini, B. (2008). Iranian Angle: Worth of Audit Report. SCMS Journal of Indian Management, 5(2), 82-90. https://www.scms.edu.in/journal/article?journal_id=38

Seaton, A. \& Montague, N. (2018). The unintended consequences of uncertainty disclosures made by auditors and managers on nonprofessional investor judgments. Accounting, Organizations and Society, (65), 44-55. https://doi.org/10.1016/j.aos.2017.10.001

Sirois, L., Bédard, J., \& Bera, P. (2018). The Informational Value of Key Audit Matters in the Auditor's Report: Evidence from an Eye-Tracking Study. Accounting Horizons 32(2), 141-162. https://doi.org/10.2308/acch-52047

Spathis, C. (2003). Audit Qualification, Firm Litigation, and Financial Information: an Empirical Analysis in Greece. International Journal of Auditing, 71 ), 71-85. https://doi.org/10.1111/1099-1123.00006

Vasgas, W. y Bayardo, J. (2013). El MILA. Mercado de integración entre Chile, Perú y Colombia. Revista de Relaciones Internacionales, Estrategia y Seguridad, 8(1), 113-133. https://doi.org/10.18359/ries.67

Villardefrancos, M., y Riviera, Z. (2006). La auditoría como proceso de control: concepto y tipología. Revista Ciencias de la información, 372), 53-59. http://cinfo.idict.cu/index.php/cinfo/article/view/90

Worthington, A., \& Higgs, H. (2006). Evaluating financial development in emerging. Journal of Economic Development, 31(1), 1-27. https://ro.uow.edu.au/commpapers/115/ 